\title{
ON SMALLEST RADICAL AND SEMI-SIMPLE CLASSES
}

\author{
by W. G. LEAVITT and J. F. WATTERS
}

(Received 18 September, 1969)

Introduction. In a recent paper [5] one of us has given a sufficient condition to be satisfied by a given property of radical classes within a universal class $\mathscr{W}$ in order that, for any subclass $\mathscr{H}$ of $\mathscr{W}$, there should be a smallest radical class having the given property and containing $\mathscr{M}$. The sufficient condition is that the class of all radical classes with the given property can be characterised as the class of all radical classes fixed by an admissible function $F$ (see Section 1 below). In this paper a necessary and sufficient condition is derived and the corresponding result for semi-simple classes is also presented. These results are given in Section 2.

In Section 3 we apply the semi-simple construction to show that, given any subclass $\mathscr{H}$ of $\mathscr{W}$, there is a largest radical $\mathscr{P}$ such that both $\mathscr{P}$ and its semi-simple class $\mathrm{s} \mathscr{P}$ are hereditary and $s \mathscr{P} \supseteq \mathscr{M}$. An example is given to show that there is, in general, no largest radical $\mathscr{P}$ such that $\mathscr{P}$ is hereditary and $s \mathscr{P} \supseteq \mathscr{M}$. Finally, in Section 4 , an example is given to show that there is, in general, no smallest radical class $\mathscr{P}$ such that $\varsigma_{\mathscr{P}}$ is hereditary and $\mathscr{P} \supseteq \mathscr{M}$.

1. Definitions. Let $\mathscr{W}$ be a universal class; that is, $\mathscr{W}$ is hereditary and homomorphically closed. Denote by $\mathscr{T}$ the class of all subclasses of $\mathscr{W}$, by $\mathscr{R}$ the class of all radical subclasses of $\mathscr{W}$, and by $\mathscr{Y}$ the class of all semi-simple subclasses of $\mathscr{W}$.

If $\mathscr{M} \in \mathscr{T}$, then a class $\mathscr{M}^{\prime} \supseteq \mathscr{M}$ is called an s-completion of $\mathscr{M}$ when $\mathscr{M}^{\prime}$ has the property:

(a) If $R \in \mathscr{M}^{\prime}$, then every non-zero ideal of $R$ has a non-zero homomorphic image in $\mathscr{M}^{\prime}$.

If $\mathscr{M}$ is an $s$-completion of itself, then we shall say that $\mathscr{M}$ is $s$-complete. We recall that every semi-simple class in $\mathscr{W}$ is $s$-complete and that if $\mathscr{M}$ is $s$-complete, then there is a smallest semi-simple class in $\mathscr{W}$, containing $\mathscr{M}[1$, p. 6]. The corresponding radical class, the upper $\mathscr{H}$-radical class, is denoted by $\mathrm{U} \mathscr{M}$. In particular, if $\mathscr{M} \in \mathscr{Y}, \mathrm{u} \mathscr{M}$ is the radical class determined by $\mathscr{H}$.

If a function $\mathrm{F}: \mathscr{R} \rightarrow \mathscr{T}$ is such that

A.1. for all $\mathscr{P} \in \mathscr{R}, \mathscr{P} \subseteq \mathrm{F} \mathscr{P}$;

A.2. if $\mathscr{P}_{1}, \mathscr{P}_{2} \in \mathscr{R}$ and $\mathscr{P}_{1} \subseteq \mathscr{P}_{2}$, then $\mathrm{F} \mathscr{P}_{1} \subseteq \mathrm{F} \mathscr{P}_{2}$;

A.3. if $\left\{\mathscr{P}_{\alpha}: \mathscr{P}_{\alpha} \in \mathscr{R}\right\}$ is defined for all ordinals $\alpha$ and $\mathscr{P}_{\alpha} \subseteq \mathscr{P}_{\beta}$ for $\alpha \leqq \beta$, then $\mathrm{F} \mathscr{P} \subseteq \bigcup \mathrm{F} \mathscr{P}_{\alpha}$, where $\mathscr{P}=\bigcup_{\alpha} \mathscr{P}_{\alpha}$;

then, following [5], $\mathrm{F}$ is said to be admissible. If a function $\mathrm{F}: \mathscr{R} \rightarrow \mathscr{T}$ satisfies A.1 and A.2 above, then we shall say that $\mathrm{F}$ is non-inductive admissible, which will be abbreviated to $n$-admissible. Note that in A.3, from all $\mathscr{P}_{\alpha} \in \mathscr{R}$ it follows without difficulty that $\mathscr{P} \in \mathscr{R}$. Also note by A.2 that A.3, in fact, implies that $\mathrm{F} \mathscr{P}=\bigcup_{\alpha} \mathrm{F} \mathscr{P}_{\alpha}$.

Here, in Theorem 1 , it is shown that, for each $\mathscr{M} \in \mathscr{T}, n$-admissibility of $\mathrm{F}: \mathscr{R} \rightarrow \mathscr{T}$ is a necessary and sufficient condition for the existence of a smallest radical class $\mathscr{M} \supseteq \mathscr{M}$ and 
such that $\mathrm{F} \mathscr{M}=\mathscr{M}$. This improves Theorem 1 of [5], where it was shown that admissibility of $\mathrm{F}$ is sufficient to imply the existence of $\mathscr{M}$.

If a function $\mathrm{F}: \mathscr{T} \rightarrow \mathscr{T}$ is such that

S.A.1. for $\mathscr{H} \in \mathscr{T}$, F $\mathscr{M}$ is an $s$-completion of $\mathscr{M}$;

S.A.2. if $\mathscr{H}_{1}, \mathscr{M}_{2} \in \mathscr{T}$ and $\mathscr{M}_{1} \subseteq \mathscr{M}_{2}$, then F $\mathscr{M}_{1} \subseteq \mathrm{F} \mathscr{M}_{2}$;

S.A.3. if $\left\{\mathscr{M}_{\alpha}: \mathscr{M}_{\alpha} \in \mathscr{Y}\right\}$ is defined for all ordinals $\alpha$ and $\mathscr{M}_{\alpha} \subseteq \mathscr{M}_{\beta}$ for $\alpha \leqq \beta$, then F $\mathscr{H} \subseteq \bigcup_{\alpha} F \mathscr{M}_{\alpha}$, where $\mathscr{M}=\bigcup_{\alpha} \mathscr{M}_{\alpha}$;

then $\mathrm{F}$ is said to be $s$-admissible. An $n s$-admissible function $\mathrm{F}: \mathscr{T} \rightarrow \mathscr{T}$ is one which satisfies S.A.1 and S.A.2. Again note that in S.A.3, from all $\mathscr{M}_{\alpha} \in \mathscr{Y}$ it follows without difficulty that $\mathscr{H} \in \mathscr{Y}$.

In [5, Theorem 2] it was shown that if $\mathrm{F}$ is an $s$-admissible function, then for each $\mathscr{M} \in \mathscr{T}$ there is a smallest semi-simple class $\mathscr{M} \supseteq \mathscr{M}$ and such that $\mathrm{F} \mathscr{M}=\mathscr{M}$. In fact, as we shall show in Theorem 2, ns-admissibility of $\mathrm{F}$ is necessary and sufficient for the existence of $\overline{\mathscr{M}}$.

We conclude this section with some remarks about our notation. If $A \in \mathscr{W}$, then $B \leqq A$ will denote that $B$ is an ideal of $A$; if $B \leqq A$ but $B \neq A$, then this will be denoted by $B<A$. Small capitals are used to denote operators on classes even though we are not usually dealing with closure operators. The lower radical class determined by $\mathscr{M} \in \mathscr{T}$ is written $\mathrm{L} \mathscr{M}$. If $\mathscr{P} \in \mathscr{R}$, then $S \mathscr{P}$ is the semi-simple class determined by $\mathscr{P}$, and $P(A)$ is the $\mathscr{P}$-radical of $A$. Finally, when the elements of a class are listed it is understood that what is meant is the class of all isomorphic copies of the rings listed.

\section{Main theorems.}

THEOREM 1. Let $\mathscr{V} \subseteq \mathscr{R}$ define a property of radical classes in $\mathscr{W}$. There exists, for each $\mathscr{M} \in \mathscr{T}$, a smallest class $\mathscr{M} \in \mathscr{V}$ with $\mathscr{M} \subseteq \mathscr{M}$ if and only if there is an n-admissible function $\mathrm{F}$ such that $\mathscr{V}=\{\mathscr{P} \in \mathscr{R}: F \mathscr{P}=\mathscr{P}\}$.

Proof. Suppose that $\mathrm{F}$ is an $n$-admissible function, $\mathscr{V}=\{\mathscr{P} \in \mathscr{R}: \mathrm{F} \mathscr{P}=\mathscr{P}\}$ and $\mathscr{M} \in \mathscr{T}$. Since the class $\mathscr{W} \in \mathscr{V}$ and $\mathscr{V} \supseteq \mathscr{M}$, there is a non-empty class $\mathscr{M}$ which is the intersection of all the classes $\mathscr{N} \in \mathscr{V}$ such that $\mathscr{N} \supseteq \mathscr{M}$. The proof in [2] that the intersection of a set of radical classes is a radical class applies also for a class of radical classes. Hence $\bar{M}$ is a radical class. By A.2, $\mathscr{M} \subseteq \mathscr{N}$ implies that $\mathrm{F} \mathscr{M} \subseteq \mathrm{F} \mathscr{N}=\mathscr{N}$ and so $\mathrm{F} \mathscr{M} \subseteq \mathscr{M}$. Therefore, from A.l, F $\mathscr{M}=\mathscr{M}$ so $\mathscr{M} \in \mathscr{V}$. Finally, if $\mathscr{K} \supseteq \mathscr{M}$ and $\mathscr{K} \in \mathscr{V}$, then $\mathscr{K} \supseteq \mathscr{M}$ and so $\overline{\mathscr{M}}$ is the smallest class in $\mathscr{V}$ which contains $\mathscr{M}$.

Conversely, if, given $\mathscr{P} \in \mathscr{R}, \mathscr{P}$ is the smallest class in $\mathscr{V}$ with $\mathscr{P} \subseteq \overline{\mathscr{P}}$, define a function $\mathrm{F}: \mathscr{R} \rightarrow \mathscr{T}$ by setting $\mathrm{F} \mathscr{P}=\bar{P}$. Then $\mathrm{F}$ is an $n$-admissible function and $\mathscr{V}=\{\mathscr{P} \in \mathscr{R}: \mathrm{F} \mathscr{P}=\mathscr{P}\}$.

REMARK 1. If $\mathrm{F}$ is an $n$-admissible function, then, setting $\mathscr{M}_{1}=\mathrm{L} \mathscr{M}$, where $\mathscr{M} \in \mathscr{T}$, and defining

$$
\mathscr{M}_{\beta}= \begin{cases}\mathrm{L}\left(\bigcup_{\alpha<\beta} \mathscr{M}_{\beta}\right) & \text { if } \quad \beta \text { is a limit ordinal, } \\ \mathrm{LF} \mathscr{M}_{\alpha} & \text { if } \beta=\alpha+1,\end{cases}
$$


we obtain an ascending chain of radical classes whose union $\mathscr{M}^{*}$ is a radical class. An easy induction argument shows that $\mathscr{M}^{*} \subseteq \mathscr{M}$ and if $\mathrm{F}$ is admissible, then $\mathscr{M}^{*}=\mathscr{M}[5$, Theorem 1]. For $n$-admissible $\mathrm{F}$ we need not have $\mathscr{M}^{*}=\mathscr{M}$ as the following example shows.

Let $\Phi_{\alpha}$ be the quotient field of the polynomial ring $\Phi\left[A_{\alpha}\right]$, where $A_{\alpha}$ is a set of commuting indeterminates of cardinality $\aleph_{\alpha}, \Phi$ is a finite field and $\alpha$ is any ordinal. The fields $\Phi_{\alpha}, \Phi_{\beta}$ have different cardinalities for $\alpha \neq \beta$, and so are non-isomorphic.

Let $\mathscr{W}=\left\{0, \Phi, \Phi_{\alpha}: \alpha\right.$ any ordinal $\}$. Every ring in $\mathscr{W}$ is simple so $\mathscr{R}=\mathscr{T}=\mathscr{Y}$. Define

and otherwise

$$
\mathrm{F}\{\mathscr{W}-\{\Phi\}\}=\mathrm{F} \mathscr{W}=\mathscr{W}
$$

$$
\mathrm{F} \mathscr{M}=\mathscr{M} \cup \Phi_{\gamma},
$$

where $\gamma$ is the least ordinal such that $\Phi_{y} \notin \mathscr{M}$. Then $\mathrm{F}$ is an $n$-admissible function which is not admissible. If $\mathscr{M}=\left\{0, \Phi_{0}\right\}$, then $\mathscr{M}^{*}=\left\{0, \Phi_{\alpha}: \alpha\right.$ any ordinal $\}$ but $\mathscr{M}=\mathscr{W}$, so $\bar{M} \neq \mathscr{M}^{*}$.

THEOREM 2. Let $\mathscr{X} \subseteq \mathscr{Y}$ define a property of semi-simple classes. There exists, for each $\mathscr{M} \in \mathscr{T}$, a smallest class $\bar{M} \in \mathscr{X}$ with $\overline{\mathscr{M}} \supseteq \mathscr{M}$ if and only if there is an ns-admissible function $\mathrm{F}$ such that $\mathscr{X}=\{\mathscr{Q} \in \mathscr{Y}: \mathrm{F} \mathscr{Q}=\mathscr{Q}\}$.

Proof. Suppose that $\mathrm{F}$ is an $n s$-admissible function $\mathscr{X}=\{\mathscr{Q} \in \mathscr{Y}: \mathrm{F} \mathscr{Q}=\mathscr{Q}\}$ and $\mathscr{M} \in \mathscr{T}$. Since $\mathscr{W} \in \mathscr{X}$ and $\mathscr{M} \subseteq \mathscr{W}$, there is a non-empty class $\mathscr{M}$ which is the intersection of all the classes $\mathscr{N} \in \mathscr{X}$ such that $\mathscr{M} \subseteq \mathscr{N}$.

By S.A.2, $\bar{M} \subseteq \mathscr{N}$ implies that $\mathrm{F} \overline{\mathscr{M}} \subseteq \mathrm{F} \mathscr{N}$ and so $\mathrm{F} \overline{\mathscr{M}} \subseteq \mathscr{M}$. Hence, from S.A.1, $\mathrm{F} \mathscr{M}=\mathscr{M}$ and $\mathscr{M}$ is s-complete. Therefore $\mathscr{M}$ satisfies condition (a) of Section 1. Furthermore, if $A \in \mathscr{W} \backslash \mathscr{M}$, there is a class $\mathscr{N} \in \mathscr{X}$ such that $\mathscr{N} \supseteq \mathscr{M}$ but $A \notin \mathscr{N}$. By the semi-simplicity of the class $\mathcal{N}$, there is $(0) \neq B \leqq A$ such that no non-zero homomorphic image of $B$ belongs to $\mathcal{N}$ and so no non-zero homomorphic image of $B$ belongs to $\mathscr{M}$. Thus $\mathscr{M}$ satisfies the condition:

(b) If every $(0) \neq B \leqq A \in \mathscr{W}$ can be mapped homomorphically onto a non-zero ring in $\overline{\mathscr{M}}$, then $A \in \mathscr{M}$.

Now any class which satisfies both (a) and (b) is semi-simple [1, Theorem 2], so $\mathscr{M} \in \mathscr{X}$ and $\mathscr{M} \supseteq \mathscr{M}$. Finally, if $\mathscr{Q} \in \mathscr{X}$ and $\mathscr{Q} \supseteq \mathscr{M}$, then $\mathscr{Q} \supseteq \mathscr{M}$ and so $\mathscr{M}$ is the smallest class in $\mathscr{X}$ which contains $\mathscr{M}$.

For the converse, define a function $\mathrm{F}: \mathscr{T} \rightarrow \mathscr{T}$ by setting $\mathrm{F} \mathscr{M}=\mathscr{M}$. Then $\mathrm{F}$ is $n s$-admissible and $\{\mathscr{Q} \in \mathscr{Y}: \mathrm{F} \mathscr{Q}=\mathscr{Q}\}=\mathscr{X}$.

ReMARK 2. If $\mathrm{F}$ is an $n s$-admissible function, then setting $\mathscr{M}_{1}=\operatorname{suF} \mathscr{M}$, where $\mathscr{H} \in \mathscr{T}$, and defining

$$
\mathscr{M}_{\beta}= \begin{cases}\bigcup_{\alpha<\beta} \mathscr{M}_{\alpha} & \text { if } \beta \text { is a limit ordinal, } \\ \operatorname{suF} \mathscr{M}_{\alpha} & \text { if } \beta=\alpha+1,\end{cases}
$$

we obtain an ascending chain of semi-simple classes whose union $\mathscr{M}^{*}$ is a semi-simple class. An easy induction argument shows that $\mathscr{M}^{*} \subseteq \mathscr{M}$ and, if $\mathrm{F}$ is $s$-admissible, $\mathscr{M}^{*}=\mathscr{M}[\mathbf{5}$, Theorem 2]. For an $n s$-admissible function $\mathrm{F}$ we need not have $\mathscr{M}^{*}=\mathscr{M}$, as the example of Remark 1 shows. 
From these improved forms of Theorems 1 and 2 of [5] it is clear that Theorems 5 and 6 of [5] can also be improved by replacing the conditions of admissibility and $s$-admissibility by $n$-admissibility and $n s$-admissibility respectively.

3. Applications of the semi-simple construction. Given $\mathscr{H} \in \mathscr{T}$, the hereditary closure of $\mathscr{H}$ is defined to be the class of all rings in $\mathscr{W}$ isomorphic to accessible subrings of $\mathscr{M}$-rings. The hereditary closure of $\mathscr{M}$ is denoted by $I \mathscr{M}$ and is the smallest hereditary class containing $\mathscr{H}$. Since every hereditary class is $s$-complete, the class $I \mathscr{H}$ is $s$-complete. A class $\mathscr{H}$ is hereditary if and only if $\mathrm{I} \mathscr{M}=\mathscr{A}$. It is easily seen that $\mathrm{I}$ is an admissible function defined on $\mathscr{T}$ and we have from Theorem 2 that, given $\mathscr{M} \in \mathscr{T}$, there is a smallest hereditary semi-simple class $\mathscr{I} \supseteq \mathscr{M}$, [4, Theorem 2].

To show that there is a smallest semi-simple class $\mathscr{J} \supseteq \mathscr{M}$ such that the radical determined by $\mathscr{J}$ is strongly hereditary, that is both $\mathscr{J}$ and its radical class are hereditary, we use a procedure which is essentially that used by Rjabuhin in [6] to construct, within the universal class of all associative rings, a largest hereditary radical whose semi-simple class contains a given class of rings.

Given $\mathscr{M} \in \mathscr{T}$, we define $J \mathscr{M}$ to be the class consisting of the ring 0 and all isomorphic copies of rings $R / J$ obtained as follows:

( $\alpha) B \leqq A \leqq R \in \mathscr{W}$,

(B) $(0) \neq A / B \in \mathscr{M}$,

( $\gamma) J \leqq R$ maximal with respect to the property $J \cap A \subseteq B$.

Then $\mathrm{J} \mathscr{H} \supseteq \mathscr{H}$ and if $\mathscr{M}_{1} \subseteq \mathscr{M}_{2}$, then $\mathrm{J} \mathscr{H}_{1} \subseteq \mathrm{J} \mathscr{M}_{2}$. In the following lemmas we establish some properties of the function $\mathrm{J}$.

LeMma 1. If $\mathscr{M}$ is s-complete, then $\mathrm{J} \mathscr{M}$ is s-complete.

Proof. Suppose that $(0) \neq I / J \leqq R / J \in J \mathscr{M}$. In the $\mathscr{M}$-ring $A / B$, where $A$ and $B$ are as in the definition of the class $\mathrm{J} \mathscr{M}$, there is the non-zero ideal

$$
\frac{(\operatorname{I\cap A} A)+B}{B} \cong \frac{\operatorname{I\cap } A}{\operatorname{I\cap } B}
$$

Therefore, since $\mathscr{M}$ is $s$-complete, there is $K<\operatorname{I\cap A}$ such that $\operatorname{I\cap B} \subseteq K$ and $(\operatorname{I\cap A}) / K \in \mathscr{M}$. Also

$$
J \cap(\operatorname{I\cap A})=\operatorname{I\cap }(J \cap A) \subseteq I \cap B \subseteq K,
$$

so there is an ideal $J^{*}$ of $I$ such that $J^{*} \supseteq J$ and $J^{*}$ is maximal with respect to the property $J * \cap(I \cap A) \subseteq K$. Since $K \neq \operatorname{I\cap A}$, the ideal $J^{*} \neq I$.

Hence, from the definition of the class $\mathrm{J} \mathscr{M}$, the ring $I / J^{*}$, which is a non-zero homomorphic image of $I / J$, is an element of $\mathrm{J} \mathscr{M}$. Thus the class $\mathrm{J} \mathscr{H}$ is $s$-complete.

LEMMa 2. Let $\mathscr{P} \in \mathscr{R}$ and $\mathrm{s} \mathscr{P}=\mathscr{M}$. If $\mathscr{P}$ is strongly hereditary, then $\mathrm{J} \mathscr{M}=\mathscr{M}$.

Proof. Let $P / J$ be the $\mathscr{P}$-radical of the ring $R / J \in \mathrm{J} \mathscr{M}$, where $B \leqq A \leqq R \in \mathscr{W}$, $(0) \neq A \mid B \in \mathscr{M}$, and $J$ is as in $(\gamma)$ above. 
Since $\mathscr{M}$ is hereditary, the ideal $((P \cap A)+B) / B$ of $A / B$ belongs to $\mathscr{M}$ and so $(P \cap A) /(P \cap B) \in \mathscr{M}$. But, because $\mathscr{P}$ is hereditary, the ideal $((P \cap A)+J) / J$ of $P / J$ belongs to $\mathscr{P}$ and so $(P \cap A) /(J \cap A) \in \mathscr{P}$. Now $J \cap A \subseteq P \cap B$, so $(P \cap A) /(P \cap B)$ is a homomorphic image of the $\mathscr{P}$-ring $(P \cap A) /(J \cap A)$. Therefore

$$
\frac{P \cap A}{P \cap B} \in \mathscr{P} \cap S \mathscr{P}
$$

and $P \cap A=P \cap B \subseteq B$. Finally, by the maximality of $J, P=J$ and $R / J \in \mathscr{M}$, which completes the proof.

LEMMA 3. Let $\mathscr{M}$ be a hereditary class such that $\mathrm{J} \mathscr{M}=\mathscr{M}$. Then $\mathscr{P}=\mathrm{U} \mathscr{H}$ is hereditary.

Proof. Let $(0) \neq A \leqq R \in \mathscr{P}$ and suppose that $A \notin \mathscr{P}$. Then there is $B \leqq A$ such that $(0) \neq A \mid B \in \mathscr{M}$. If $J \leqq R$ and is maximal with respect to the property $J \cap A \subseteq B$, then $R / J \in \mathrm{J} \mathscr{M}=\mathscr{M} \subseteq \mathrm{S} \mathscr{P}$. But $R / J \in \mathscr{P}$, so $R=J$, which implies that $A=B$. This is a contradiction, so $A \in \mathscr{P}$ and $\mathscr{P}$ is hereditary.

With the aid of these lemmas and Theorem 2, we are now able to prove the main result of this section.

THEOREM 3. Given $\mathscr{M} \in \mathscr{T}$, there is a smallest semi-simple class $\mathscr{J} \supseteq \mathscr{M}$ such that the radical determined by $\mathscr{J}$ is strongly hereditary.

Proof. Let $\mathscr{X}$ be the class of all semi-simple classes whose corresponding radicals are strongly hereditary. Given $\mathscr{M} \in \mathscr{T}$, we define $\mathrm{F} \mathscr{M}=\mathrm{J}(\mathrm{I} \mathscr{M})$. It is immediate from Lemma 1 that $\mathrm{F}$ is an $n s$-admissible function. Put

$$
\mathscr{X}^{\prime}=\{\mathscr{Q} \in \mathscr{Y}: \mathrm{F} \mathscr{Q}=\mathscr{Q}\} .
$$

From Lemma $2, \mathscr{X}^{\prime} \supseteq \mathscr{X}$. On the other hand, if $\mathscr{Q} \in \mathscr{X}^{\prime}$ and $\mathscr{P}$ is its corresponding radical class, then both $\mathrm{I} \mathscr{Q}=\mathscr{Q}$ and $\mathrm{s} \mathscr{Q}=\mathscr{Q}$. Hence $\mathscr{Q}$ is a hereditary class such that $\mathrm{J} \mathscr{Q}=\mathscr{Q}$ and then, from Lemma $3, \mathrm{u} \mathscr{Q}=\mathscr{P}$ is also hereditary. Thus $\mathscr{P}$ is a strongly hereditary radical and $\mathscr{Q} \in \mathscr{X}$. Therefore $\mathscr{X}^{\prime}=\mathscr{X}$ and the result follows from Theorem 2.

It might be conjectured that, given $\mathscr{M} \in \mathscr{T}$, there is a smallest semi-simple class $\mathscr{Q} \supseteq \mathscr{M}$ and such that the radical determined by $\mathscr{Q}$ is hereditary. This is false as the following example shows.

EXAMPLE 1. Let $K$ be the algebra over $G F(p)$ with generators $e, x, y, z$ and multiplication determined by the table:

\begin{tabular}{|c|c|c|c|c|}
\hline & $e$ & $x$ & $y$ & $z$ \\
\hline$e$ & $e$ & $e$ & $e$ & $x$ \\
\hline$x$ & $e$ & 0 & 0 & $e$ \\
\hline$y$ & $e$ & 0 & $y$ & $z$ \\
\hline$z$ & $x$ & $e$ & 0 & $y$ \\
\hline
\end{tabular}


Let $E$ be the subring generated by $e$ and $X$ the subring generated by $x$ and $e$. Then $E \leqq X \leqq K$ and $X$ is the only ideal in $K$ other than (0) and $K$ itself. The rings $X / E, K / X$ and $E$ are non-isomorphic and simple.

Let $\mathscr{W}=\{K, X, E, K|X, X| E, 0\}, \mathscr{M}=\{0, K\}, \mathscr{Q}_{1}=\{0, K, X, E\}$ and $\mathscr{Q}_{2}=\{0, K, X \mid E\}$. Then $\mathscr{Q}_{1}$ and $\mathscr{Q}_{2}$ are minimal $s$-completions of $\mathscr{M}$ so, in general, there is not a smallest $s$-completion of a class $\mathscr{M}$. This confirms a conjecture made in [4]. Furthermore $\mathscr{Q}_{\mathbf{1}}$ and $\mathscr{Q}_{2}$ are the semi-simple classes determined respectively by the radical classes $\{0, K|X, X| E\}$ and $\{0, E, K / X\}$, each of these being hereditary.

4. Smallest radicals. It is known [3, Corollary] that, given $\mathscr{M} \in \mathscr{T}$, there is a smallest hereditary radical class $\mathscr{H} \supseteq \mathscr{M}$ and a smallest strongly hereditary radical class $\mathscr{D} \supseteq \mathscr{M}$. The existence of these radicals can be established from Theorem 1 using the $n$-admissible functions I and IG, where G, as in [5], is given by

$$
\mathrm{G} \mathscr{P}=\{K \in \mathscr{W}: J \leqq I \leqq A \in \mathscr{W} \text { with } J \in \mathscr{P} \text { and } K \text { the ideal of } A \text { generated by } J\}
$$

and $\mathscr{P} \in \mathscr{R}$. The function $\mathrm{G}$ is itself $n$-admissible, and it is not difficult to see that $\mathrm{G} \mathscr{P}=\mathscr{P}$ if and only if, given $I \leqq A \in \mathscr{W}$, we have $P(I) \leqq A$. We shall denote the smallest such radical class containing $\mathscr{M} \in \mathscr{T}$ by $\mathscr{G}$. It is clear that $\mathscr{H} \subseteq \mathscr{D}$ and, from [1, Lemmas 68 and 69], $\mathscr{G} \subseteq \mathscr{D}$. All three radicals are, in general, distinct as the next example shows.

EXAMPLE 2. By a construction of Rjabuhin [7] there are rings $A_{1}, A_{2}, A_{3}, A_{4}$ such that the only proper ideal of $A_{i+1}$ is $A_{i}$ for $i=1,2,3$. Also $A_{1}$ and $B_{i+1}=A_{i+1} / A_{i}$ are non-isomorphic simple rings.

Let $\mathscr{W}=\left\{0, A_{1}, A_{2}, A_{3}, A_{4}, B_{2}, B_{3}, B_{4}\right\}, \mathscr{M}=\left\{0, A_{2}, B_{2}\right\}, G=\left\{0, A_{2}, A_{3}, B_{2}, B_{3}\right\}$ and $\mathscr{H}=\left\{0, A_{1}, A_{2}, B_{2}\right\}$. Then $\mathscr{W}$ is a universal class, $\mathscr{H}$ is a radical class in $\mathscr{W}, \mathscr{H}$ is the smallest hereditary radical class containing $\mathscr{M}, \mathscr{G}$ is the smallest radical class containing $\mathscr{M}$ and such that, given $I \leqq A \in \mathscr{W}$, we have $G(I) \leqq A$, and $\mathscr{H} \cup \mathscr{G}=\mathscr{D}$ is the smallest strongly hereditary radical containing $\mathscr{M}$. Each of these assertions is easily checked so we omit the proofs.

The semi-simple classes corresponding to the radical classes $\mathscr{G}$ and $\mathscr{D}$ are always hereditary and again one might conjecture that, given $\mathscr{M} \in \mathscr{T}$, there is a smallest radical class $\mathscr{P} \supseteq \mathscr{M}$ with hereditary semi-simple class. Using Example 1, we can show that this is, in general, false. Let $\mathscr{W}$ be as in Example $1, \mathscr{M}=\{0, E\}, \mathscr{P}_{1}=\{0, X, E, X / E\}$ and $\mathscr{P}_{2}=\{0, E, K, K / X\}$. Then $\mathscr{M}$ is a radical class in $\mathscr{W}$ and both $\mathscr{P}_{1}$ and $\mathscr{P}_{2}$ are minimal radical classes containing $\mathscr{M}$ and having hereditary semi-simple class. Since $\mathscr{P}_{1}$ and $\mathscr{P}_{2}$ are incomparable, there is no smallest radical containing $\mathscr{H}$ and having hereditary semi-simple class. Again each of these assertions is easily checked and the proofs are omitted.

\section{REFERENCES}

1. N. Divinsky, Rings and Radicals (London, 1965).

2. W. G. Leavitt, Sets of radical classes, Publ. Math. Debrecen 14 (1967), 321-324.

3. W. G. Leavitt, Strongly hereditary radicals, Proc. Amer. Math. Soc. 21 (1969), 703-705.

4. W. G. Leavitt, Hereditary semi-simple classes, Glasgow Math. J. 11 (1970), 7-8. 
5. W. G. Leavitt, Radical and semi-simple classes with specified properties, Proc. Amer. Math. Soc. 24 (1970), 680-687.

6. Ju. M. Rjabuhin, On the imbedding of radicals, Isv. Akad. Nauk. Mold. SS 11 (1963), 34-40.

7. Ju. M. Rjabuhin, Lower radicals of rings, Mat. Zametki 2 (1967), 239-244.

UNIVERSITY OF NEBRASKA

The UNIVERSITY, LeICESTER

LINCOLN, NEBRASKA

U.S.A. 